\title{
Setting plasma parameters for automated interplanetary shocks detection
}

Victória Fernandes do Amaral ${ }^{* 1,3}$, Manilo Soares Marques ${ }^{1}$, Ezequiel Echer ${ }^{2},{ }^{1}$ UFRN, ${ }^{2}$ INPE, ${ }^{3}$ Bolsista IC/INPE

Copyright 2021, SBGf - Sociedade Brasileira de Geofísica.

This paper was prepared for presentation during the $17^{\text {th }}$ International Congress of the Brazilian Geophysical Society held in Rio de Janeiro, Brazil, 8-11 November 2021.

Contents of this paper were reviewed by the Technical Committee of the $17^{\text {th }}$ International Congress of the Brazilian Geophysical Society and do not necessarily represent any position of the SBGf, its officers or members. Electronic reproduction or storage of any part of this paper for commercial purposes without the written consent of the Brazilian Geophysical Society is prohibited.

\begin{abstract}
Society's technological advancement, marked by the use of artificial satellites, requires a better understanding of Sun-Earth interactions. The solar wind and associated events, such as coronal mass ejections and corotating interaction regions, directly affect Earth's magnetosphere. One of the consequences of these interactions is the sudden changes in the plasma parameters - the density, temperature and speed of the protons, as well as the total magnetic field measurement; this region of discontinuity is called interplanetary shock. The study's purpose is to improve the parameters for the automatic detection of shocks by the algorithm proposed by Cash et al., 2014. For the identification of fast forward shock, there is a sudden increase in the values of all plasma parameters at the same instance of time. For the algorithm to notice this signature, a directional derivative was performed with the average of the pre-shock and post-shock values, with a time interval of 5 minutes. The directional derivative quantitatively informs the gain in the parameter value and, in order to better values for the parameters when compared to the original algorithm, a total of 140 shocks occurred in solar cycle 23 , i.e., between the years 1998 and 2007, were analyzed. After the analysis, there was an increase of $50 \%$ in density, $6 \%$ in speed, $4 \%$ in temperature. The magnetic field showed an increase of $31 \%$ when combined with the other parameters, while of $120 \%$ separately. For the efficacy test, the shocks that occurred in 2014 were visually identified, and then a comparison was performed between the list of shocks found automatically with the original parameters and those incorporated in this work. The result was a 16\% increase in the hit rate, a $49 \%$ increase in the value, an $8 \%$ increase in the probability of detection and a $21 \%$ decrease in the false positive rate. The results of the study indicate that resetting the parameters values proved to be efficient and renders automatic detection more accurate, allowing to perform the analysis of years of data with greater speed and precision.
\end{abstract}

Keywords: interplanetary shocks. algorithm. automated shock detection. fast forward shock. 